Background Current management of seizures at the end of life utilises sedating medications (midazolam and phenobarbital) administered via the subcutaneous route. The subcutaneous administration of levetiracetam presents the possibility of managing seizures at the end of life without causing sedation.

Methods A comprehensive literature review was performed and this data was combined with data from a prospective observational audit undertaken in a regional palliative care network. A search of EMBASE, Medline, CINAHL, ClinicalTrials.gov and the WHO International Trials Registry for "subcutaneous AND levetiracetam" or "subcutaneous AND keppra" or "levetiracetam SC" on the 16 th of July 2015 and updated the search on the 2nd of August 2016.

Results 7 papers were identified from the literature review; 4 case reports and 3 observational case series; reporting on a total of 53 cases where subcutaneous levetiracetam was administered.

We report 20 further cases of subcutaneous levetiracetam administration from a prospective observational case series. Doses ranged from $250 \mathrm{mg}-4000 \mathrm{mg}$ daily. Oral to subcutaneous conversion ratios where stated were 1:1. Levetiracetam was reported as the sole administered anti-epileptic drug (AED) in 8 cases and no seizures were reported until death in 5 cases. 5 were switched back to enteral Levetiracetam. In 7 cases, Levetiracetam was combined with AEDs to provide seizure control at the end of life. There was one report of a sterile abscess after 25 days of continuous subcutaneous administration.

Conclusions A combined analysis of 73 reported cases world wide of subcutaneous levetiratcetam administration suggests it may have a role in the management of seizures at the end of life. However randomised controlled trials are urgently needed to establish the efficacy and tolerability of subcutaneous levetiracetam administration. If proven to be safe and effective subcutaneous levetiracetam offers the potential to prevent and treat seizures without causing unnecessary sedation at the end of life.

\section{P-64 QUALITY IMPROVEMENT PROJECT- END OF LIFE MEDICATION USE}

${ }^{2}$ Jeffrey Kong, ${ }^{2}$ Ben Harris. 'St Richard's Hospital, Chichester, UK; ${ }^{2}$ Isle of Man Noble Hospital, UK

\subsection{6/bmjspcare-2017-00133.64}

Background Palliative care is a multidisciplinary approach to specialised medical care for patients with terminal illnesses, mainly providing relief from symptoms, physical and mental stress of an illness. There are multiple components to managing patients in terminal phase. Anticipatory prescribing of as required medication in advance is one of them. The significance lies in ensuring no delay in responding to a symptom. This retrospective audit was conducted to assess compliance with local guidelines.

Methods Medical records of inpatient Deaths during November and December 2014 were requested and data specifically, medications prescription, was collected. There should be 4 prescriptions with clearly stated route, frequency, indication and maximum dose.

Results Out of 56 patients included in the audit, only 25 patients received end of life care. 24 patients were included as 1 chart was unavailable. Only $74 \%$ patients had their regular medication discontinued. The most common choice of documentation was palliation (34\%) followed by 'keep comfortable' (29\%), with minority using phrases like 'T.L.C', 'stop all treatment'. The audit revealed relatively satisfactory targets for respective prescriptions with the highest $87 \%$ for pain followed by $85 \%$ for nausea, $78 \%$ for agitation and dyspnea and lastly, 74\% for respiratory secretion. Only a small number of Syringe driver was used - mainly pain relief with only $17 \%$. Opioid Conversion is rather poor. Only 57\% of the patients previously on regular pain relief had accurate dosing following the conversion chart.

Conclusion To ensure anticipatory medication being accurately prescribed, end of life prescription chart would be introduced with opioid conversion chart included. Healthcare professionals should be given teaching and made aware of the chart and hospice service. Palliative care team should review all patients as soon as they are commenced on palliative care however this would not feasible as patients in hospital setting tend to die within days.

\section{P-65 TITLE: MULTI-WARD HOSPICE EXCHANGE PROGRAM}

${ }^{1} J u d e$ Edwards, ${ }^{2}$ Susan Heatley. ${ }^{1}$ St Ann's Hospice, Cheadle, UK; ${ }^{2}$ Manchester Royal Infirmary, Central Manchester University Hospitals

\subsection{6/bmjspcare-2017-00133.65}

An exchange program has existed between qualified nurses at St Ann's Hospice and Renal Departments in partnership trusts for over 10 years, the outcomes of which have always been positive and have allowed all staff to develop their knowledge, skills and confidence. With this in mind it was decided to expand the program to include the Cardiology, Haematology and Respiratory departments. The diversity of the acute settings addresses the changing nature of hospice care where patients are admitted with complex clinical reasons, and presents hospices with significant challenges for education and training.

The provision for palliative care is inconsistent across the country, this is particularly true of out of hours. Although it is unfair to directly compare hospices and hospitals, the advantages of sharing specific knowledge and skills across the 2 is clear.

A one week nurse exchange; centred on observations, is agreed between St Ann's Hospice and the Renal, Cardiology, Haematology and Respiratory departments of Central Manchester Foundation Trust. Discussions are underway to include Gastro-enterologly. The program adopts a hands on approach where knowledge and skills are shared through shadowing and observations.

Evaluation Following on from the renal exchange program last year, a new evaluation form has been produced that captures the data in a more quantitative manner, thus allowing outcomes to be more auditable.

Overall Objectives 1. Equip St Ann's staff with the skills and knowledge to deal with more clinically complex patients

2. Raise awareness of individualised end of life care in a hospice setting

The Future This is an ongoing project, aiming to cascade across all hospital specialties, with a view to develop EoLC 'Champions' in each specialty. Our goal would be to see this partnership program replicated in other organisations. 